\title{
Exogenous follistatin administration ameliorates cisplatin- induced acute kidney injury through anti-inflammation and anti-apoptosis effects
}

\author{
Koken E $E^{1}$ Oz Oyar E², Uyanikgil Y³, Azak Pazarlar B², Bilister C², Aksun $\mathrm{S}^{4}$, \\ Yigitturk $\mathrm{G}^{5}$, Koken $\mathrm{EC}^{6}$
}

Department of Physiology, Faculty of Medicine, Afyonkarahisar University of Health Sciences, Afyon, Turkey. dr.ebrukoken@gmail.com

\begin{abstract}
OBJECTIVES: This study was aimed to explore the effects of follistatin on cisplatin-induced renal dysfunction, histopathological changes, apoptosis, inflammation and oxidative damage in rats.

BACKGROUND: Follistatin plays an important role in the developmental and regeneration processes of kidney by blocking the actions of activin, which is a member of transforming growth factor- $\beta$ superfamily. METHODS: Twenty seven rats were separated into 4 equal groups: Control, $\mathrm{Cp}$ (cisplatin, $6 \mathrm{mg} / \mathrm{kg}$, intrapertoneally (ip)), F1 (cisplatin $+1 \mu \mathrm{g} /$ day follistatin ip for 4 consecutive days) and F4 (cisplatin $+4 \mu \mathrm{g} /$ day follistatin ip single dose) groups. Renal health was monitored by blood urea nitrogen, serum creatinine and histological analysis. Apoptosis, inflammation and oxidative stress was investigated in kidney tissue. Activin A levels in serum and kidney were evaluated as well.

RESULTS: Follistatin administration showed a considerable nephroprotective effect against cisplatin-induced nephrotoxicity by preventing renal functional and structural abnormalities, apoptosis and inflammation. The activin A levels in both serum and kidney were also suppressed by follistatin administration.

CONCLUSION: Exogenous follistatin ameliorates acute kidney injury, by blocking activin A. The renoprotective effect of follistatin against cisplatin-induced nephrotoxicity appears to be associated with its anti-inflammatory, antiapoptotic and direct nephroprotective actions (Tab. 1, Fig. 7, Ref. 23). Text in PDF www.elis.sk.

KEY WORDS: activin, acute kidney injury, cisplatin, follistatin, nephroprotection.
\end{abstract}

\section{Introductions}

Cisplatin is an inorganic platinum based chemotherapeutic drug, used for the treatment of various kinds of solid tumors. Nephrotoxicity is the major side-effect of cisplatin, which occurs in $20 \%$ of patients receiving cisplatin treatment (1). Despite the

\begin{tabular}{l}
\hline${ }^{1}$ Department of Physiology, Faculty of Medicine, Afyonkarahisar Univer- \\
sity of Health Sciences, Afyon, Turkey, ${ }^{2}$ Department of Physiology, Faculty \\
of Medicine, Izmir Katip Celebi University, Izmir, Turkey, ${ }^{3}$ Department \\
of Histology and Embryology, Cord Blood, Cell and Tissue Research and \\
Application Centre, Faculty of Medicine, Ege University, Izmir, Turkey, \\
${ }^{4}$ Department of Biochemistry, Izmir Katip Celebi University Faculty of \\
Medicine, Izmir, Turkey, ${ }^{5}$ Department of Histology and Embryology, Fa- \\
culty of Medicine, Mugla Sitki Kocman University, Mugla, Turkey, and \\
${ }^{6}$ Department of Biophysics, Institute of Health Sciences, Aydin Adnan \\
Menderes University, Aydin, Turkey
\end{tabular}

Address for correspondence: E. Koken, MD, Department of Physiology, Faculty of Medicine, Afyonkarahisar University of Health Sciences, 03211, Afyon, Turkey. Phone: +90.555 .6274834$

Acknowledgement: Authors declared that chemical agents and consumables used in this study were supplied from the project funded by Izmir Katip Celebi University, Scientific Research Foundation with project number 2017-TDU-TIPF-0032. underlying molecular mechanism of cisplatin-induced nephrotoxicity is complex and remains uncertain, activation of inflammatory pathways, induction of oxidative stress damage and renal tubular cell death were demonstrated in kidney tissues of cisplatin treated animals (2). In order to be successful in preventing cisplatin damage, it is necessary to target these multiple mechanisms, without reducing the effect of cisplatin against cancer cells.

Follistatin is a multifunctional regulatory protein, secreted by many tissues (3). The primary function of follistatin is mainly related with its capability of binding activins, members of transforming growth factor- $\beta$ (TGF- $\beta$ ) superfamily (4). Follistatin is expressed on the surface of the target cells of activins in two main forms consisting of 288 (FS288) and 315 (FS315) amino acids. FS288 is more potent than FS315 in its ability to bind activin to the target cell surface (5). Activins trapped irreversibly by follistatin are endocytosed into the cell and inactivated by proteolysis (6). Activins are basically in two forms according to the type of $\beta$-subunit found in their structure: activin A and activin B. Although it is known that both activin species are involved in activin-follistatin interaction, studies were focused more on activin A, since follistatin has a higher affinity for activin $\mathrm{A}(7)$. 
143-150
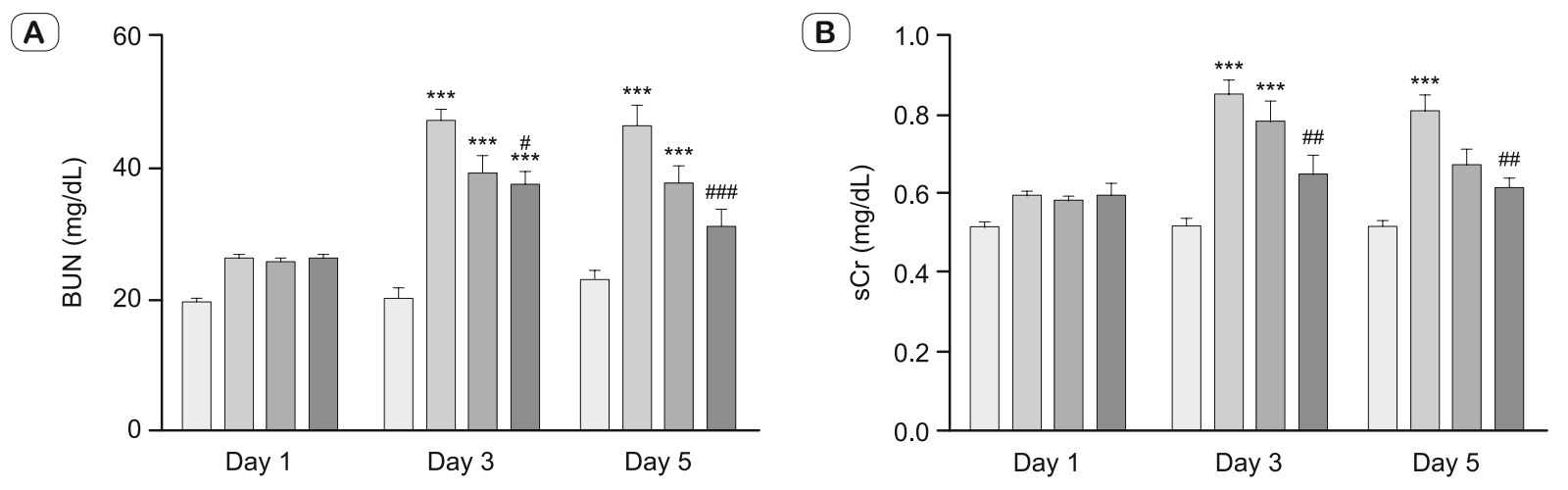

$\square$ F4 $\square$ F1 $\square \mathrm{Cp} \quad \square$ Control

Fig. 1. Effects of follistatin on serum urea and creatinine levels in rats with cisplatin-induced nephrotoxicity. (A) BUN; (B) sCr. Data were expressed as the means \pm standard error of the mean (SEM) $(n=7$ in each group). All experiments were duplicated three times. Statistically significant differences were determined by two-way ANOVA, ${ }^{* * *} \mathbf{p}<0.001$ versus control group; ${ }^{\#} p<0.05,{ }^{\#} p<0.01,{ }^{\# \#} p<0.001$ versus cisplatin alone treated group. $B U N$ blood urea nitrogen, $s C r$ serum creatinine. $C p$ Cisplatin group, F1 Cisplatin + Follistatin $1 \mu$ g - 4 day group, F4 Cisplatin + Follistatin $4 \mu \mathrm{g}$ - single day group
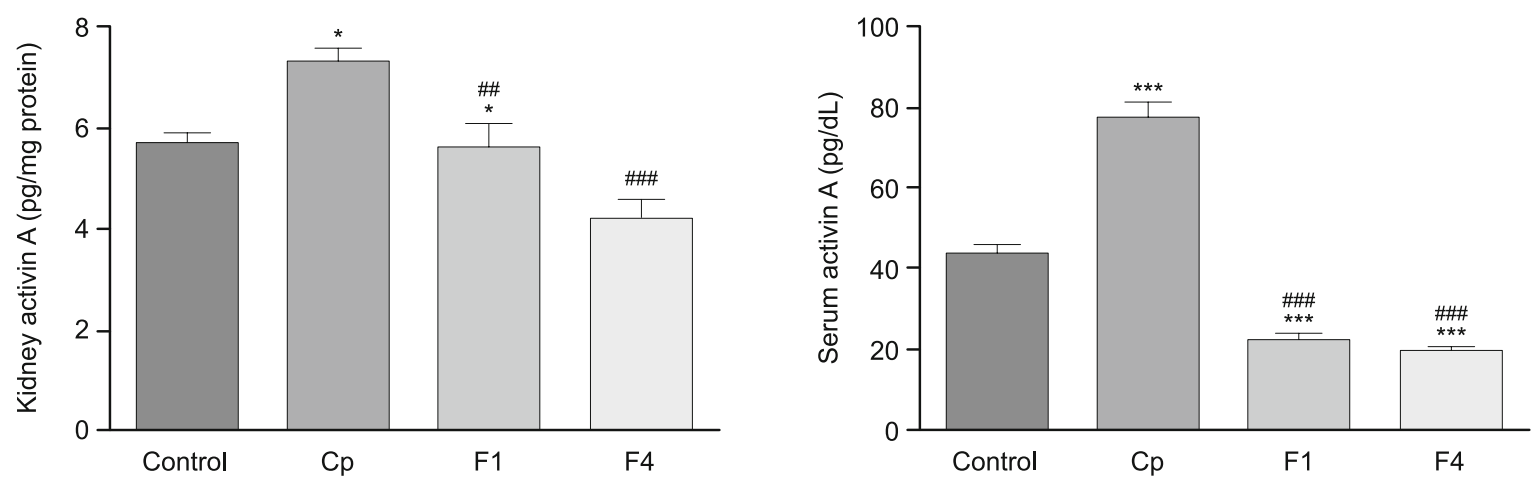

Fig. 2. Effects of follistatin on Activin A levels in kidney tissue and serum in rats with cisplatin-induced nephrotoxicity. (A) Kidney Activin A; (B) Serum Activin A. The levels of activin A in kidney tissue and serum were measured by ELISA. Data were expressed as the means \pm SEM $(n=7$ in each group). All experiments were duplicated three times. Statistically significant differences were determined by one-way ANOVA, ${ }^{*} \mathbf{p}<0.05,{ }^{* *} \mathbf{p}<0.01,{ }^{* * *} \mathrm{p}<0.001$ versus control group; ${ }^{\# \#} \mathbf{p}<0.01,{ }^{\# \#} \mathbf{p}<0.001$ versus cisplatine alone treated group. ELISA enzyme-linkedimmunosorbent-assay. For other abbreviations, see Fig. 1

Tab. 1. Effects of follistatin on histopathological findings in kidney tissues of cisplatin-treated rats.

\begin{tabular}{lcccc}
\hline Histopathological findings & Control & $\mathrm{Cp}$ & $\mathrm{F} 1$ & $\mathrm{~F} 4$ \\
\hline Necrosis of renal tubules & - & +++ & ++ & + \\
Degeneration of podocytes and edema & - & ++ & + & + \\
Vacuolisation in proximale tubules & - & +++ & ++ & + \\
Vascular dilatation and congestion & - & +++ & ++ & + \\
Inflammatory cell infiltration & $\mathrm{n}$ & +++ & ++ & + \\
Hyaline casts & $\mathrm{n}$ & +++ & ++ & ++ \\
\hline
\end{tabular}

$\mathrm{n}=$ normal; $-=$ no alteration; $+=10-25 \%$ mild altered tubules; $++=25$ to $50 \%$ moderate altered tubules; $+++=$ more than $50 \%$ severe altered tubules.

Cp Cisplatin group, F1 Cisplatin + Follistatin $1 \mu \mathrm{g}-4$ day group, F4 Cisplatin + Follistatin $4 \mu \mathrm{g}$ - single day group

The activin-follistatin system is an important regulatory system modulating developmental and regeneration processes of the kidney. Follistatin induces kidney development in embryogenesis and promotes tubular regeneration after renal injury by blocking acitivin A $(8,9)$. Follistatin has also regulatory roles in several inflammatory and immune processes. Many studies showed that activin A promoted inflammation by stimulating production of inflammatory mediators including tumor necrosis factor- $\alpha$ (TNF- $\alpha$ ), interleukin- 6 and $1 \beta$ (IL-6, IL-1 $\beta$ ), and nitric oxide, whereas follistatin reduced oxidative stress and modulated the inflammatory process in tissue repair by neutralizing activin A $(10,11)$. Since all these regulatory properties of follistatin are involved in the pathogenesis of cisplatin nephrotoxicity, it could be anticipated that follistatin administration would improve kidney recovery after cisplatin-induced acute kidney injury.

This is the first study to evaluate the anti-inflammatory, antiapoptotic and antioxidant effect of follistatin on cisplatin-induced nephrotoxicity. Inhibition of activin by exogenous follistatin administration may be a new treatment approach for acute renal injury induced by cisplatin. 

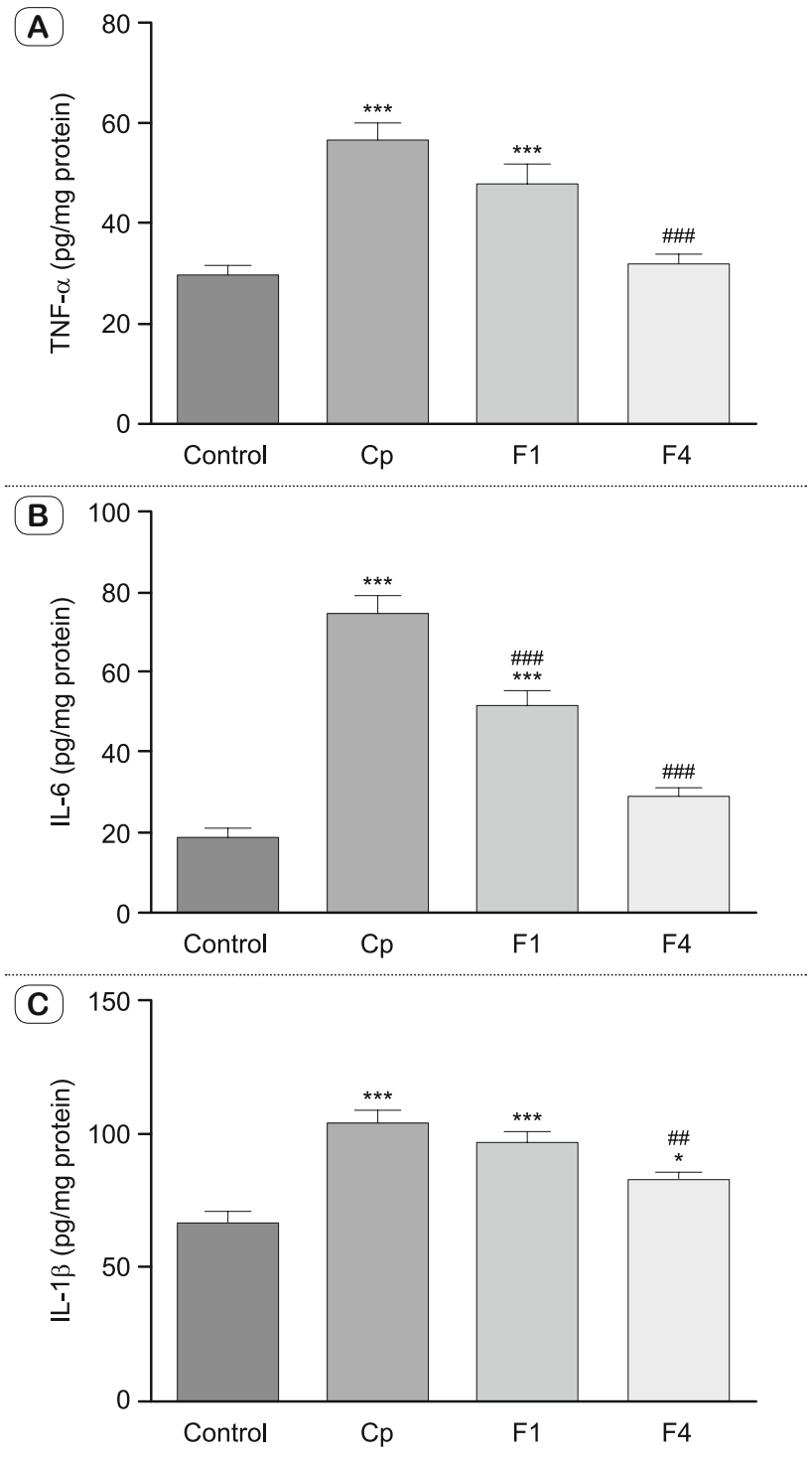

Fig. 3. Effects of follistatin on inflammatory cytokines production in kidney tissues of cisplatin treated rats. (A) TNF- $\alpha$; (B) IL-6; (C) IL-

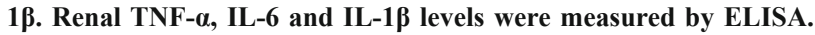
Data were expressed as the means \pm SEM $(n=7$ in each group). All experiments were duplicated three times. Statistically significant differences were determined by one-way ANOVA, $* * * p<0.001$ versus control group; ${ }^{\#} \mathbf{p}<\mathbf{0 . 0 1},{ }^{\# \#} \mathbf{p}<0.001$ versus cisplatin alone treated group. TNF- $\alpha$ tumor necrosis factor- $\alpha(T N F-\alpha), I L-6$ interleukin-6, $I L-1 \beta$ interleukin-1 $\beta$. For other abbreviations, see previous figures.

\section{Materials and methods}

Animals

Adult male Wistar Albino rats weighting $310 \pm 24$ grams were allowed to acclimatize for 7 days prior to experimentation. Rats were group housed (seven rats in a cage) under 12:12 h light/dark cycles at room temperature $\left(24 \pm 1^{\circ} \mathrm{C}\right)$ and a relative humidity of $50 \pm 10 \%$ with an access to food and water ad libitum. All experimental procedures were approved by Ethics Committee for Animal Experiments in Ege University (Approval number 2017-27)
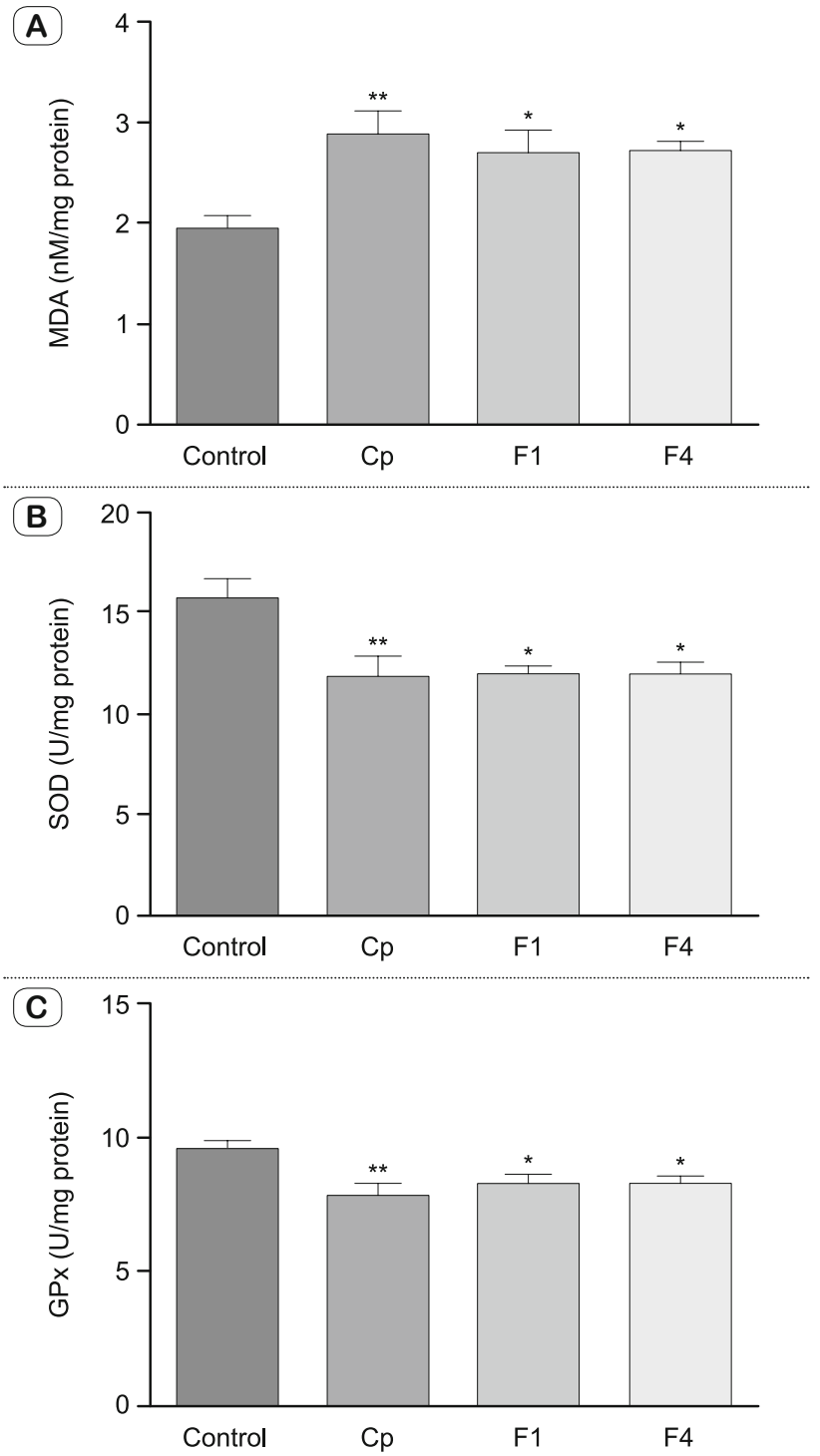

Fig. 4. Effects of follistatin on changes of oxidative biomarkers in kidney tissues of cisplatin-treated rats. (A) MDA levels, (B) SOD activities (C) GPx activities. Renal MDA levels, SOD activities and GPx activities were determined spectrophotometrically. Data were expressed as the means \pm SEM $(n=7$ in each group). All experiments were duplicated three times. Statistically significant differences were determined by one-way ANOVA, * $\mathbf{p}<\mathbf{0 . 0 5}, * * \mathbf{p}<0.01$ versus control group. $M D A$ malondialdehyde, $S O D$ superoxide dismutase, GPx glutathione peroxidase. For other abbreviations, see Fig. 1.

and carried out in accordance with the guidelines of the European Communities Council Directive (86/609/EEC).

\section{Experimental design}

Cisplatin (P4394) was purchased from Sigma-Aldrich Co. (St. Louis, MO, USA), and recombinant human Follistatin 288 (5836FS-025) was purchased from R\&D Systems (Minneapolis, MN, USA). All other chemicals used in this study were of analytical grade and obtained from common commercial sources. 

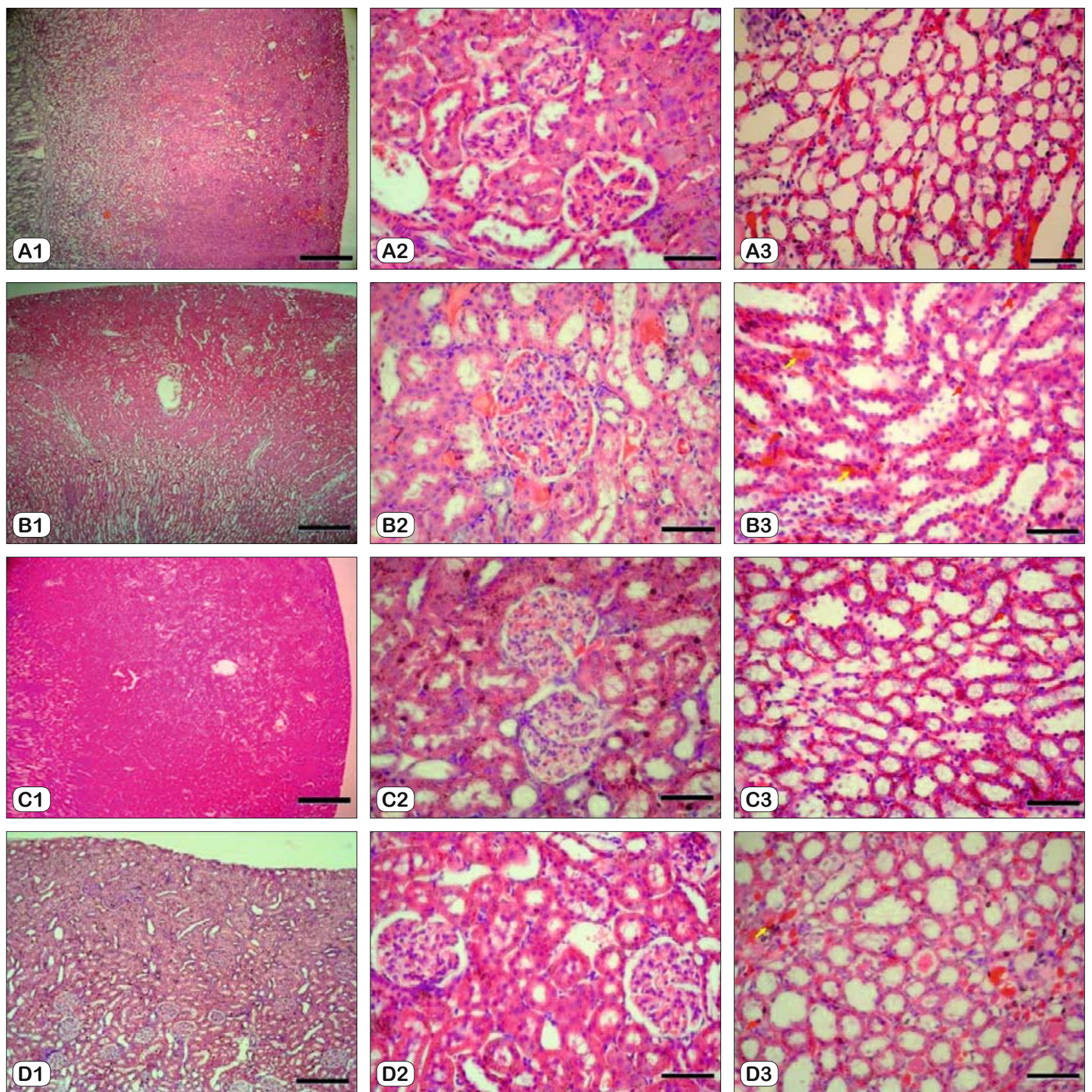

Fig. 5. Effects of follistatin on renal tubular damage in kidney tissues of cisplatin-treated rats (H\&E staining). (A) Control group; (B) Cp group; (C) F1 group; (D) F4 group. Representative Hematoxylin and Eosin stained sections from indicated groups. Series A is 4x, B and C are 40x magnification, respectively; scale bar is $1250 \mu \mathrm{M}$ for $4 \mathrm{x}$ and $125 \mu \mathrm{M}$ for $40 \mathrm{x}$ magnification. Red arrow heads indicate tubular necrosis and degeneration, yellow arrow heads indicate vascular congestion. $H \& E$ Hematoxylin-Eosin staining. For other abbreviations, see Fig. 1.

The dose cisplatin $(12,13)$ and follistatin $(14,15)$ used in this study was chosen based on previous reports. The rats were grouped as follows ( $\mathrm{n}=7$ /group):

1. Control group: Rats were not administered any treatment.

2. Cisplatin (Cp) group: Cisplatin $(6 \mathrm{mg} / \mathrm{kg}$, i.p. $)$ was injected at day 0 .

3. F1 group: Cisplatin was administrated as in the Cp group. 24 hours after cisplatin injection, the rats were administered follistatin $(1 \mu \mathrm{g} / \mathrm{rat}$, i.p. $)$ daily for four days.
4. F4 group: Cisplatin was administrated as in the Cp group. 24 hours after cisplatin injection, the rats were administered single dose of follistatin ( $4 \mu \mathrm{g} / \mathrm{rat}$, i.p.).

On days 1 and 3, blood samples were taken from the tail vein and centrifuged at $1,000 \times \mathrm{g}$ for 15 minutes at $4{ }^{\circ} \mathrm{C}$. The obtained serums were used to measure blood urea nitrogen (BUN) and serum creatinine $(\mathrm{sCr})$. On the 5 th day, animals were anesthetized with sodium pentobarbital $(150 \mathrm{mg} / \mathrm{kg}$, i.p. $)$ and were killed by 

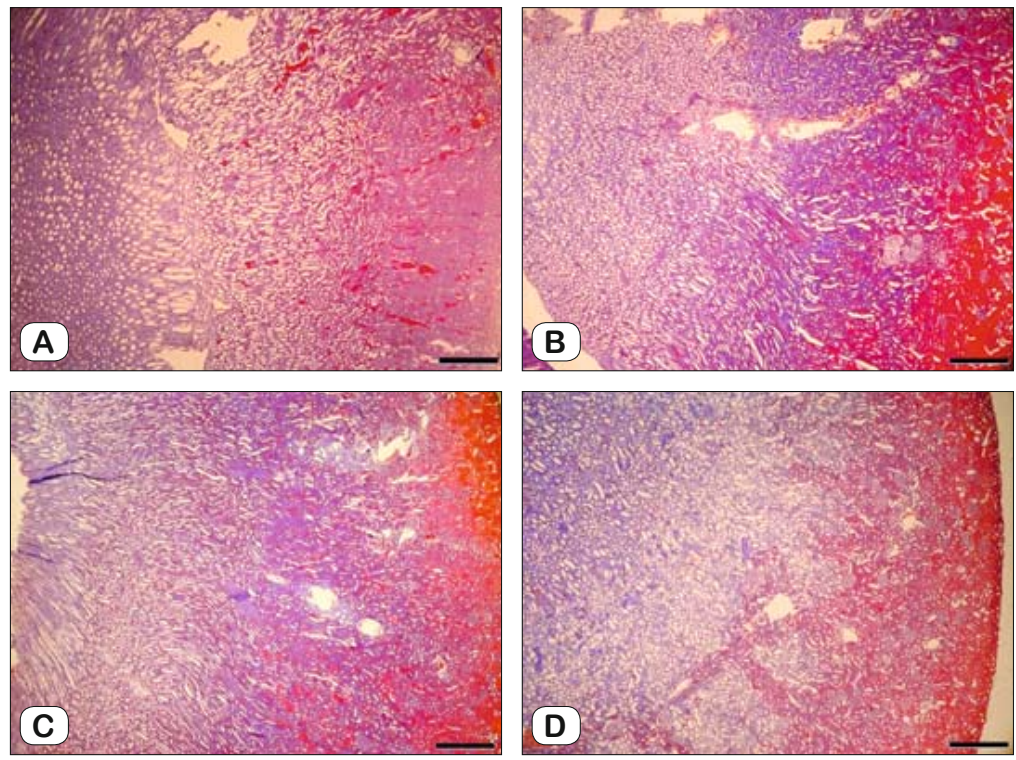

Fig. 6. Effects of follistatin on renal tubular damage in kidney tissues of cisplatin-treated rats (M-A staining). (A) Control group; (B) Cp group; (C) F1 group; (D) F4 group. Representative Mallory-Azan stained sections (4x) from indicated groups. Scale bar is $1250 \mu \mathrm{M}$ for $4 \mathrm{x} . M-A$ Mallory-Azan staining. For other abbreviations, see Fig. 1.

assay (ELISA) kit (E-EL-R0001, Elabscience; Houston, TX, USA), according to the manufacturer's instructions, using an absorbance reader (BioTek ELx808, BioTek; Winooski, VT, USA) at $450 \mathrm{~nm}$. Results were calculated by the fourparameter curve method and expressed as $\mathrm{pg} / \mathrm{mg}$ tissue protein and $\mathrm{pg} / \mathrm{mL}$ serum.

\section{Measurement of proinflammatory cytokine levels in kidney tissue}

The concentrations of TNF- $\alpha$ (BMS622), IL-6 (BMS625) and IL-1 $\beta$ (BMS630) were determined with rat ELISA kits (Invitrogen; CA, USA), according to the manufacturer's instructions, using an absorbance reader at $450 \mathrm{~nm}$. Results were calculated by the four-parameter curve method and expressed as pg/mg tissue protein.

Measurement of oxidative stress markers in kidney tissues

The concentration of MDA, a product of lipid peroxidation, was determined spectrophotometrically by measuring color produced

cardiac exsanguination. Blood samples taken from the heart were centrifuged as previously described and the serum was used for estimation of $\mathrm{BUN}, \mathrm{sCr}$ and activin A levels. Both the kidneys were dissected at the time of sacrifice, right kidneys from all rats were snap frozen, preserved at $-80^{\circ} \mathrm{C}$ and used for biochemical estimations. Left kidneys were preserved in $10 \%$ neutral buffered formalin for histological analyses.

\section{Detection of renal function}

The renal function was evaluated by assessing the serum levels of BUN and sCr using a standard autoanalyzer (Architect c4000 AutoAnalyzer, Abbott Diagnostics, MA, USA). The results were expressed as $\mathrm{mg} / \mathrm{dL}$.

\section{Preparation of kidney tissue homogenate}

Kidney tissues were homogenized in Tissue Extraction Reagent (Invitrogen; CA, USA) containing a Protease Inhibitor Cocktail Tablet (Roche; Indianapolis, IN) according to the kit protocol to measure TNF- $\alpha$, IL-6, IL- $1 \beta$, superoxide dismutase (SOD), glutathione peroxidase (GPx) and activin A levels. For determination of malondialdehyde (MDA) level, tissues were homogenized in $0.1 \mathrm{M}$ phosphate buffer (1:5). All the homogenates were centrifuged at $10,000 \mathrm{xg}$ for 15 minutes at $4^{\circ} \mathrm{C}$ and the supernatants were removed, vortexed, aliquoted, and stored at $-80^{\circ} \mathrm{C}$ until use. Protein concentrations of tissue homogenates were determined using the same autoanalyzer.

\section{Measurement of activin A concentrations in serum and in kidney tissue}

Activin A concentrations were evaluated in kidney tissue homogenate and in serum by rat enzyme-linked immunosorbent in the reaction of MDA with thiobarbituric acid according to Draper and Hadley and the results were expressed as $\mathrm{nmol} / \mathrm{mL}$ (16). The activity of SOD was evaluated by the spectrophotometric method (17) using the RANSOD kit (Randox Labs; Crumlin, UK). GPx activity was determined by the spectrophotometric method according to Paglia and Valentine (18) using the RANSEL kit (Randox Labs; Crumlin, UK). The activity of GPx and SOD in kidney tissue was then calculated per milligram of tissue and expressed in international units of kidney tissue activity $\mathrm{U} / \mathrm{mg}$.

\section{Histological analysis of kidney}

Formalin fixed renal sections $(5 \mu \mathrm{m})$ were stained with hematoxylin-eosin (H\&E) and mallory-azan (M-E) according to standard methods. The sections were examined and photographed with Olympus C-5050 digital camera (Olympus; Tokyo, Japan) mounted on Olympus BX51 microscope. Morphological analysis was assessed by computerized image analysis system (Image-ProExpress 1.4.5, Media Cybernetics Inc. Rockville, MD, USA). Histopathological changes were evaluated as previously reported (19).

Apoptotic cells were detected using the TdT-mediated dUTP nick-end labeling (TUNEL) assay kit (In Situ Cell Death Detection Kit, POD, Roche Diagnostics GmbH; Mannheim, Germany) as described by manufacturer's protocol. Stained slides were photographed and TUNEL-positive cells were quantified by counting brown-colored cells in 10 fields with $\mathrm{x} 40$ objective.

\section{Statistical analysis}

The Graphpad Prism 6 graphical and statistics package (Graph-Pad Software Inc.; San Diego, CA, USA) was used for 
presentation and analyses of the data. Results were expressed as the mean \pm standard error of the mean (SEM). Two-way ANOVA repeated measurements were used to evaluate significant changes of BUN and $\mathrm{sCr}$ levels between the groups, and during different days of study respective prior time point in each group. Bonferroni post hoc test was used as a correction for multiple comparison. The rest of the data were statistically analyzed using one way ANOVA, followed by Tukey's multiple comparison test. Differences with $\mathrm{p}$ values of 0.05 or lower were considered statistically significant.

\section{Results}

Effects of follistatin on Cp-induced renal dysfunction

Cisplatin treatment induced significant increases in BUN $(\mathrm{p}<0.001)$ and $\mathrm{sCr}(\mathrm{p}<0.001)$ at day 3 and 5 compared to the control group, reflecting the successful establishment of acute kidney injury. At the dose $4 \mu \mathrm{g}$ follistatin nearly reversed serum urea and creatinine to normal levels at day 5 (Fig. 1).

Effects of Cp and follistatin on activin A levels

Cisplatin significantly elevated the activin A levels in serum $(p<0.05)$ and in the kidney tissue $(p<0.001)$ compared to the control group, whereas follistatin treatments depressed both (Figs $2 \mathrm{~A}$ and $2 \mathrm{~B}$ ).

Effects of follistatin on Cp-induced inflammation

Compared to control, cisplatin treatment evoked significant increases in renal TNF- $\alpha(\mathrm{p}$ $<0.001)$, IL-6 $(\mathrm{p}<0.001)$ and IL-1 $\beta(\mathrm{p}<0.001)$ production (Figs 3A, 3B and 3C). Follistatin treatment at high dose $(4 \mu \mathrm{g})$ significantly $(\mathrm{p}$ $<0.001$ : TNF- $\alpha$ and IL-6; $p<0.01$ : IL-1 $\beta$ ) restored the level of these proinflammatory cytokines, when compared to cisplatin group (Fig. 3). Though follistatin treatment at low dose $(1 \mu \mathrm{g})$ restored the level of IL- 6 , the levels of TNF- $\alpha$ and IL- $1 \beta$ remain unaltered, when compared to those of cisplatin alone treated rats.
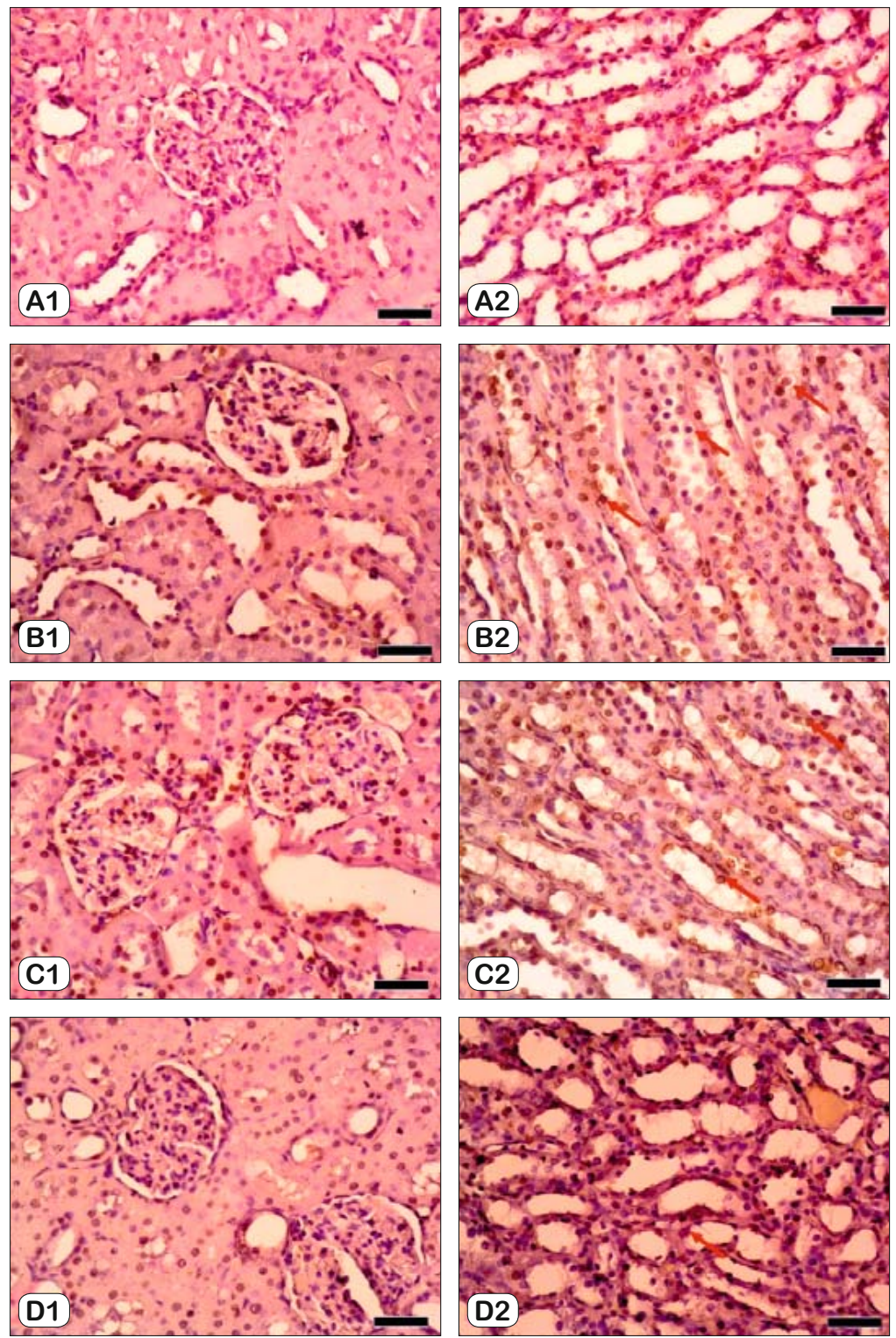

Fig. 7. Effects of follistatin on apoptosis in kidney tissues of cisplatin-treated rats. (A) Control group; (B) Cp group; (C) F1 group; (D) F4 group. Representative TUNEL stained sections $(x 40)$ from indicated groups. Red arrow heads indicate TUNEL positive nuclei. Scale bar is $125 \mu \mathrm{M}$ for $\times 40$. TUNEL TdT-mediated dUTP nick-end labeling. For other abbreviations, see Fig. 1.

\section{Effects of follistatin on Cp-induced oxidative stress}

Cisplatin administration induced a statistically significant increase $(\mathrm{p}<0.01)$ in renal MDA formation, in contrast to the control group. Follistatin treatment caused a non-significant elevation in MDA levels ( $p>0.05)$ when compared with the cisplatin-treated group (Fig 4A). SOD and GPx activities in kidney tissues were significantly suppressed by cisplatin treatment in contrast to the control group $(\mathrm{p}<0.01)$. Follistatin administration increased antioxidant enzyme activities compared to the $\mathrm{Cp}$ group; however it was not significant $(\mathrm{p}>0.05)$ (Figs $4 \mathrm{~B}$ and $4 \mathrm{C}$ ).

\section{Effects of follistatin on Cp-induced histopathological changes}

The examined kidney sections of the control group revealed normal structure, normal glomerular, tubular, interstitial and vascular architecture with no sign of inflammatory cells (Tab. 1, Fig. 5A). While the cisplatin group demonstrated an extensive degeneration, inflammatory cell infiltration, interstitial cell edema vascular congestion, hyaline cast and desquamation of tubular epithelium with a loss of normal architecture were observed (Fig. 5B). All these phenomena caused by cisplatin treatment were dose-dependently suppressed by follistatin adminis- 
tration (Tab. 1, Figs 5C and 5D). When the dose of follistatin was $4 \mu \mathrm{g}$, the histological alterations of kidney tissues resulting from cisplatin nephrotoxicity were almost completely ameliorated (Fig. 5D).

Figure 6 shows the presence of intense M-A staining in the kidney sections of the Cp group, especially in the cortex regions (Fig. 6B). This staining image was slightly reduced in the F1 group and significantly decreased in the F4 group (Figs 6C and 6D).

\section{Effects of follistatin on Cp-induced apoptotic changes}

The number of TUNEL-positive cells in kidney sections was significantly increased after cisplatin treatment compared to the number of the control group (Fig. 7B). Follistatin administration at $1 \mu \mathrm{g}$ decreased the number of cisplatin-induced TUNEL-positive cells (Fig. 7C). The extent of cell death was markedly reduced in rats that had received $4 \mu \mathrm{g}$ follistatin treatment as compared to Cp group (Fig. 7D).

\section{Discussion}

The present study investigated for the first time the beneficial effect of follistatin on cisplatin-induced acute kidney injury. In agreement with previous studies, we found that a single intraperitoneal injection of cisplatin $(6 \mathrm{mg} / \mathrm{kg})$ resulted in an impaired glomerular function and renal tubular damage manifested in elevated BUN and $\mathrm{sCr}$ (Fig. 1) and histopathological damage with tubular degeneration and tubular necrosis in kidneys (Fig. 5). Furthermore, proinflammatory biomarkers (TNF- $\alpha$, IL- 6 and IL1 $\beta$ ) and lipid peroxidation product (MDA) levels were elevated, the antioxidant enzym (SOD and GPx) levels decreased and apoptosis of renal tubular cells was induced in cisplatin treated rats.

At high dose $(4 \mu \mathrm{g})$, follistatin substantially suppressed cis platin-induced renal disfunction, tubular damage and local inflammation in kidneys and effectively attenuated apoptotic changes of renal tubular cells. However, follistatin did not show a sufficient effect to reduce the oxidative damage after cisplatin in the kidneys. These findings indicate that treatment with follistatin can confer a protective effect against cisplatin-induced acute kidney injury through anti-inflammatory and antiapoptotic mechanisms.

Maeshima et al (15) and Fang et al (20) reported that follistatin treatment prevented the histologic changes, reduced renal function biomarkers and apoptosis in tubular cell and suppressed systemic inflammatory response in rodent model of renal ischemia-reperfusion injury. The results of the previously mentioned studies and our data suggest that nephroprotective effects of follistatin may be due to its anti-inflammatory and anti-apoptotic activity. The possible efficacy of follistatin to reduce oxidative damage in acute kidney injury was first investigated in this study.

Many studies have shown that activins played an important role in inflammation and oxidative damage, and serum and tissue activin levels were increased in oxidative stress and several inflammatory conditions $(21,22)$. In this study, we measured the levels of activin A and showed that the activin A increased after cisplatin nephrotoxicity compared to the control group, whereas follistatin treatment decreased activin A in both serum and kidney after an acute kidney injury. Unlike our findings, Fang et al (20) reported that follistatin treatment had no impact on renal activin levels and its renoprotective effect could be predominantly due to suppression of the systemic inflammatory response. In our study, we found that follistatin caused a decrease in activin A levels in both serum and kidneys. Considering the local anti-inflammatory and potent regenerative effects of follistatin in the aforementioned results, we suggest that follistatin may exhibit its nephroprotective effects not only through systemic effects but also local mechanisms.

In conclusion, according to the findings of the present study, follistatin ameliorated tubular damage and apoptotic cell death in renal tissues, inhibited the formation of proinflammatory cytokines, lipid peroxidation products and restored antioxidant enzyme activity in the kidney. The results of our study showed that treatment with follistatin produced a significant protection against nephrotoxicity induced by cisplatin. Furthermore, the beneficial effect of follistatin as evidenced by biochemical findings and supported by histological findings in cisplatin toxicity might be due to the anti-inflammatory, antiapoptotic and antioxidative effect of this protein.

Consequently, exogenous follistatin administration may prevent acute kidney injury induced by cisplatin by blocking activin A activity. Besides, as shown in previous studies (23), the possible anti-cancer effect of follistatin can make it an ideal agent for the treatment of cisplatin-induced acute kidney injury, as it does not inhibit the chemotherapeutic efficacy of cisplatin while preventing nephrotoxicity.

\section{References}

1. Yao X, Panichpisal K, Kurtzman N, Nugent K. Cisplatin nephrotoxicity: A review. Am J Med Sci 2007; 334 (2): 115-124.

2. Pabla N, Dong Z. Cisplatin nephrotoxicity: Mechanisms and renoprotective strategies. Kidney Int 2008; 73 (9): 994-1007.

3. Kogawa K, Ogawa K, Hayashi Y, et al. Immunohistochemical localization of follistatin in rat tissues. Endocrinol Jpn 1991; 38 (4): 383-391.

4. Shimonaka M, Inouye S, Shimasaki S, Ling N. Follistatin binds to both activin and inhibin through the common beta-subunit. Endocrinology 1991; 128 (6): 3313-3315.

5. Lerch TF, Shimasaki S, Woodruff TK, Jardetzky TS. Structural and biophysical coupling of heparin and activin binding to follistatin isoform functions. J Biol Chem 2007; 282: 15930-15939.

6. Hashimoto O, Nakamura T, Shoji H, et al. A novel role of follistatin, an activin-binding protein, in the inhibition of activin action in rat pituitary cells: Endocytotic degradation of activin and its acceleration by Follistatin associated with cell-surface heparan sulfate. J Biol Chem 1997; 272: $13835-13842$.

7. Hedger MP, De Kretser DM. The activins and their binding protein, follistatin-Diagnostic and therapeutic targets in inflammatory disease and fibrosis. Cytokine Growth Factor Rev 2013; 24 (3): 285-295.

8. Maeshima A, Nojima Y, Kojima I. The role of the activin-follistatin system in the developmental and regeneration processes of the kidney. Cytokine Growth Factor Rev 2001; 12 (4): 289-298. 


\section{3-150}

9. Kojima I, Maeshima A, Zhang YQ. Role of the activin-follistatin system in the morphogenesis and regeneration of the renal tubules. Mol Cell Endocrinol 2001; 180 (1-2): 179-182.

10. Harrison CA, Gray PC, Vale WW, Robertson DM. Antagonists of activin signaling: Mechanisms and potential biological applications. Trends Endocrinol Metab 2005; 16 (2): 73-78.

11. Westall GP, Snell GI, Loskot M, et al. Activin Biology After Lung Transplantation. Transplant direct 2017; 3 (6): e159.

12. Liu Q, Hu S, He Y, et al. The protective effects of Zhen-Wu-Tang against cisplatin-induced acute kidney injury in rats. PLoS One 2017; 12(6): e0179137.

13. Helmy MM, Helmy MW, Abd Allah DM, et al. Selective ET(A) receptor blockade protects against cisplatin-induced acute renal failure in male rats. Eur J Pharmacol 2014; 730: 133-139.

14. Maeshima A, Mishima K, Yamashita S, et al. Follistatin, an activin antagonist, ameliorates renal interstitial fibrosis in a rat model of unilateral ureteral obstruction. Biomed Res Int 2014; 2014 (376191): 1-10.

15. Maeshima a, Zhang YQ, Nojima Y, et al. Involvement of the activinfollistatin system in tubular regeneration after renal ischemia in rats. J Am Soc Nephrol 2001; 12 (8): 1685-1695.

16. Draper HH, Hadley M. Malondialdehyde determination as index of lipid Peroxidation. Methods Enzymol 1990; 186: 421-431.
17. Woolliams JA, Wiener G, Anderson PH, McMurray CH. Variation in the activities of glutathione peroxidase and superoxide dismutase and in the concentration of copper in the blood in various breed crosses of sheep. Res Vet Sci 1983; 34 (3): 253-256.

18. Paglia DE, Valentine WN. Studies on the quantitative and qualitative characterization of erythrocyte glutathione peroxidase. J Lab Clin Med 1967; 70 (1): 158-169.

19. Uyanikgil Y, Ateș U, Baka M, et al. Immunohistochemical and histopathological evaluation of 2,4-dichlorophenoxyacetic acid-induced changes in rat kidney cortex. Bull Environ Contam Toxicol 2009; 82 (6): 749-755.

20. Fang DYP, Lu B, Hayward S, et al. The Role of Activin A and B and the Benefit of Follistatin Treatment in Renal Ischemia-Reperfusion Injury in Mice. Transplant direct 2016; 2 (7): e87.

21. Mandang S, Manuelpillai U, Wallace EM. Oxidative stress increases placental and endothelial cell activin A secretion. J Endocrinol 2007; 192 (3): 485-493.

22. Hedger MP, Winnall WR, Phillips DJ, de Kretser DM. The Regulation and Functions of Activin and Follistatin in Inflammation and Immunity. Vitam Horm 2011; 85: 255-297.

23. Shi L, Resaul J, Owen S, et al. Clinical and Therapeutic Implications of Follistatin in Solid Tumors. Cancer Genomics Proteomics 2016; 13 (6): 425-435. 